
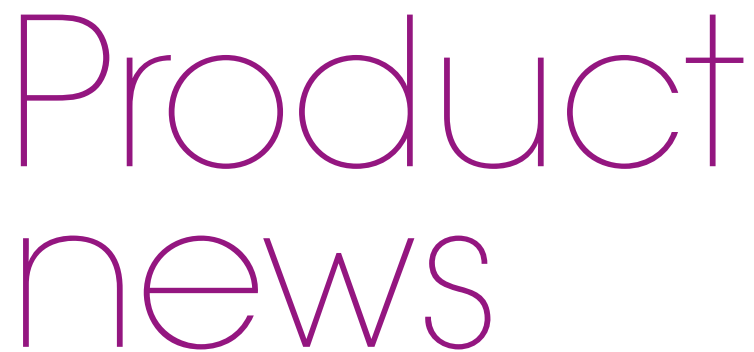

Product news is provided as a service to readers using text and images from the manufacturer, supplier or distributor and does not imply endorsement by BDJ Team. Normal and prudent research should be exercised before purchase or use of any product mentioned.

\section{NEW HANDPIECE DECONTAMINATION PROTOCOL}

As the UK's market leader in handpiece and decontamination technology, NSK is committed to improving the standards of handpiece decontamination, especially in light of the heightened risks posed by transmission of harmful pathogens, particularly by asymptomatic patients.

Until now, the risk from dental handpieces has tended to focus on the issues surrounding aerosol production. However, little has been said about the importance of the need to correctly decontaminate handpieces which is vital in helping to prevent the risk of crossinfection between patients.

NSK's SMART3 handpiece

decontamination protocol consists of three key steps - Cleaning, Lubrication \& Sterilisation - and, when using a proven, automated system, provides the following benefits:

- SAFER - compared with inconsistent manual processes and the use of incorrect autoclave/sterilisation cycles

- MEASURED - employing proven systems that dose the correct volumes of cleaning solution and lubricant, handpieces can be sterilised using the correct autoclave cycles - S or B type vacuum

- AFFORDABLE - ensures the overall

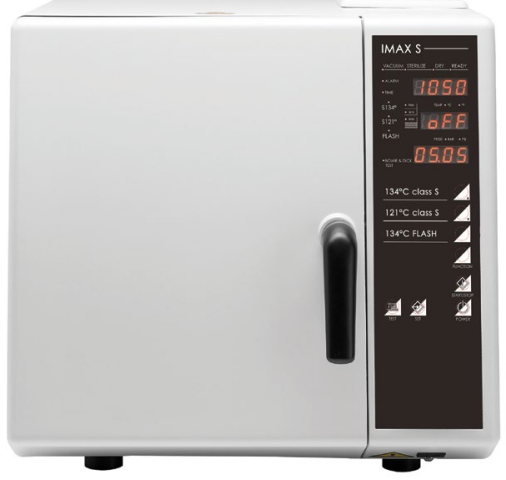

process will deliver a return on investment based on the cost savings of the consumables used, over-reliance on expensive inventory and, ongoing handpiece maintenance costs

- REPRODUCIBLE - provides consistent results, time after time, by removing the ambiguities associated with a manual process

- TIME SAVING - significantly decreases the time required to manually decontaminate handpieces, helping improve throughput and reducing costly delays in the process.

To discover more about NSK's wide range of decontamination equipment and the NSK SMART3 protocol visit mynsk. co.uk/smart3/.

\title{
SAVE TIME, MONEY AND STRESS
}

Help future proof your practice by outsourcing payroll to premium provider Wagemate.

It doesn't matter if your practice is big or small, you will save time, money and stress with Wagemate.

Everyone will be paid correctly, and on time. You can focus on developing your business, with peace of mind that if you need to upscale your payroll, or the rules change, there is no problem that the experts at Wagemate cannot solve, and fast!

Imagine payroll taken off your hands, with you staying fully compliant - with Wagemate you'll be one step ahead. Call today to find out how.

To better manage your payroll, contact payroll specialists Wagemate today, tel: 03330 102102 or email info@wagemate.com.
SUSTAINABLE DENTAL INSTRUMENTS

Dental manufacturer Young Innovations is kicking off autumn with a 'Go Green' campaign. The focus is on the American Eagle Quik-Tip portfolio.

The handles of the eco-friendly prophylaxis instruments are combined with exchangeable tips. This reduces waste, costs and conserves resources because only dull working ends are discarded instead of the entire instrument. The special design guarantees consistent quality at all times.

Take advantage of a great offer: Buy ten Quik-Tips, trade in ten old instruments and receive four new Quik-Tip Instruments free. The offer is valid for all XP Technology and Talon Tough Quik-Tips until the end of November 2021.

Quik-Tip handles are available in three types and multiple colours, allowing customisation. This simplifies, for example, identification by room, user and area of application. Quik-Tips are available for scalers, curettes and Gracey Curettes, either with Talon Tough stainless steel or XP Technology. The XP alloy makes the working ends extremely hard and resistant. They always retain their sharp cutting edges and don't require resharpening. The Pro Thin option with extra fine tips guarantees optimal access to deep periodontal pockets and exceptionally gentle treatment.

All Quik-Tip attachments can be exchanged quickly using the accompanying tool. Complete instruments can be easily processed in the washer-disinfector. For more information about Quik-Tips, visit www.am-eagle.org.
If you would like to promote your products or services direct to the dental industry in BDJ Team, call Andy May on 02078434785 or emaila.may@nature.com 